Revista Colombiana de Obstetricia y Ginecología Vol. 57 No. 3 • 2006 • (211-215)

Reporte de CAso

\title{
MANEJO DE LOS SÍNTOMAS DOLOROSOS DE LA ENDOMETRIOSIS CON DISPOSITIVO INTRAUTERINO DE LEVONORGESTREL EN UNA PACIENTE CON PELVIS CONGELADA: REPORTE DE CASO
}

Managing endometriosis pain symptoms with a levonorgestrel intrauterine device in a patient suffering firom firozen pelvis: a case report

José Fernando De Los Ríos, M.D. *; Erika Molina, M.D.**

Recibido: abril 17/06 - Revisado: julio 31/06 - Aceptado: agosto 3/06

\section{RESUMEN}

El manejo de los síntomas dolorosos de la endometriosis es un tema controvertido en nuestra especialidad. La mayoría de los tratamientos utilizados ofrecen diferentes grados de mejoría a las pacientes mientras los reciben, tienen múltiples efectos secundarios y es previsible una recaída pronto en el tiempo siguiente a su descontinuación. Muchos de los medicamentos utilizados son costosos y dados sus efectos adversos no deben ser utilizados por mucho tiempo. En algunas pacientes con paridad satisfecha la anexo-histerectomía es una alternativa terapéutica para mejorar la calidad de vida cuando han sufrido la enfermedad durante toda su etapa reproductiva. En casos avanzados de endometriosis, dicho tipo de cirugía puede ser difícil y poner en riesgo de daño a los órganos pélvicos. Describimos el caso de una paciente en quien fue imposible practicar la

* Ginecólogo Endoscopista, Hospital Pablo Tobón Uribe, Profesor adscrito de Ginecología Universidad Pontificia Bolivariana, Medellín, Colombia Correo electrónico: cristidelos@epm.net.co

Dirección: carrera 50 A \# 64 - 65. Consultorio 203. Clínica del Prado. Medellín, Colombia. Teléfono: (574) 2119843 - (574) 3215029 Fax: (574) 2119844

** Residente II de Ginecología y Obstetricia Universidad Pontificia Bolivariana, Medellín, Colombia. cirugía optándose por manejar sus síntomas con un dispositivo intrauterino de levonorgestrel (Mirena ${ }^{\circledR}$ ) con un resultado satisfactorio.

Palabras clave: endometriosis severa, pelvis congelada, dispositivo intrauterino liberador de levonorgestrel, Mirena ${ }^{\circledR}$, tratamiento médico.

\section{SUMMARY}

Medical management of pain in patients suffering endometriosis is a controversial topic in gynaecology. Most regimens offer patients different degrees of improvement whilst they are on them but they are accompanied by many adverse effects and there will probably be a recurrence of pain after they have been discontinued. Most treatment is expensive and should not be taken for long periods of time due to concomitant adverse effects. Some patients, having no further wish to bear children, will benefit from hysterectomy with bilateral salpingo-oophorectomy. Such surgery can improve their quality of life after suffering from pain throughout their reproductive years. Surgery can be very difficult in advanced endometriosis and can become dangerous for other pelvic organs. The case of a patient in which surgery was impossible 
due to a frozen pelvis is described here. Successful symptom control was finally achieved by choosing to manage her pain by applying a levonorgestrel intrauterine device (Mirena).

Key words: severe endometriosis, frozen pelvis, levonorgestrel intrauterine device, Miren ${ }^{\circledR}$, medical treatment.

\section{INTRODUCCIÓN}

El manejo médico de la endometriosis avanzada y sintomática, es un tema controversial en ginecología. La manipulación hormonal de los focos de endometrio ectópico tan solo disminuye su actividad pero no los elimina, no siendo por lo tanto una terapia ablativa, sino un tratamiento sintomático, cuyos beneficios solo se notan mientras la paciente reciba la medicación. Una vez suspendida, en menos de 1 año, hasta el $40 \%$ de las mujeres presentarán recurrencia de su sintomatología. ${ }^{1}$

Dentro de las herramientas terapéuticas disponibles para el manejo de la endometriosis, los análogos de la GnRH considerados como el "Gold standard", ${ }^{2}$ son costosos y poseen muchos efectos secundarios que limitan su uso por períodos de tiempo prolongados. De hecho, el estudio reportado que por más tiempo los ha usado es de 12 meses. ${ }^{3}$ Otros medicamentos como el danazol también poseen efectos adversos marcados, derivados de su acción androgénica. Los progestágenos son un grupo amplio de medicamentos que se han utilizado con esta indicación a diferentes concentraciones y por diversas vías de administración. Su uso ha demostrado beneficios en el manejo de estas pacientes, incluso siendo comparables los análogos de la GnRH. ${ }^{4}$

En pacientes con enfermedad avanzada y sintomática con paridad satisfecha, una de las opciones terapéuticas es la anexo-histerectomía, procedimiento que provee un alto porcentaje de mejoría al anular por completo el influjo hormonal proveniente de los ovarios y al extirpar el órgano proveedor de reflujo de tejido endometrial a la cavidad pélvica. Sin embargo, la toma de esta decisión no siempre es fácil especialmente en mujeres jóvenes en quienes siempre deberá valorarse juiciosamente el riesgo contra el beneficio de una menopausia quirúrgica con todas sus consecuencias. Especialmente importante es informar a las pacientes que una histerectomía sin la remoción de los ovarios, aunque podría ser un procedimiento que mejore su calidad de vida y disminuya sus síntomas, deja la posibilidad de continuar con dolor hasta en un 62\% de los casos, mientras apenas un $10 \%$ de las pacientes continuarán sintomáticas cuando se realiza la ooforectomía bilateral. Por otra parte el 31\% de las pacientes en quienes se deje tejido ovárico, requerirán nueva cirugía en el transcurso de su vida, mientras apenas necesitarán reintervención el 3,7\% de aquellas en quienes se remuevan ambas gónadas. ${ }^{5}$

En raros casos, donde la enfermedad es inoperable o cuando el riesgo quirúrgico excede los beneficios esperados, necesitamos retornar al manejo médico con las limitaciones que ya hemos descrito, especialmente la de que no contamos con medicamentos tolerables ni probados en el largo plazo.

Se ha sugerido que el dispositivo intrauterino liberador de Levonorgestrel podría tener un papel importante en el manejo sintomático de estas pacientes. En 1997, se publica una serie de casos de pacientes con diagnóstico imagenológico y clínico de adenomiosis en quienes se controlaron la menorragia y la dismenorrea con la aplicación de levonorgestrel por medio de un dispositivo intrauterino. ${ }^{6}$ Con este trabajo, se abrió la puerta para la investigación de esta sustancia aplicada por esta ruta en enfermedades pélvicas semejantes como es el caso de la endometriosis. Desde entonces, varios estudios intentan valorar el papel que podría tener el dispositivo intrauterino liberador de levonorgestrel en el manejo de la endometriosis.

El objetivo de este artículo es reportar la evolución de la sintomatología de una paciente con 
endometriosis manejada con un dispositivo intrauterino liberador de levonorgestrel (Mirena ${ }^{\circledR}$ ), en quien no fue posible llevar a cabo una cirugía definitiva, dados los hallazgos intraoperatorios de una pelvis congelada y la negativa razonable de la paciente y su familia a exponerse a los riesgos quirúrgicos que un procedimiento de tal magnitud le imponía.

\section{DESCRIPCIÓN DEL CASO}

Mujer de 38 años de edad, casada, con dos hijos quien consultó al Hospital Pablo Tobón Uribe de Medellín, Colombia, institución de cuarto nivel de complejidad cuyo servicio de ginecología atiende pacientes con contraindicaciones para ser manejadas en los niveles inferiores del sistema. Acudió con un cuadro de dolor abdominal intenso, localizado en ambas fosas ilíacas. Relataba una historia de múltiples consultas al servicio de urgencias por presentar episodios de dolor semejantes, dismenorrea severa incapacitante, asociada en ocasiones a otros síntomas como vómito, diarrea y dispareunia profunda. Había recibido múltiples tratamientos con anti-inflamatorios no esteroideos, analgésicos y anti-espasmódicos sin mejoría. Refería ciclos menstruales regulares con sangrado moderado. Un año antes de la consulta, se le practicó ligadura de varicocele pélvico y durante esta intervención los cirujanos vasculares describieron la presencia de endometriosis y síndrome adherencial pélvico. No tenía otros antecedentes de importancia. Al examen físico se encontró una paciente con 82 latidos cardíacos por minuto y una presión arterial de 125/80 mmHg. Había dolor a la palpación profunda de todo el hemiabdomen inferior, pero no se evidenciaron masas. El examen vaginal reveló un útero en anteversoflexión, fijo, levemente doloroso al intentar movilizarlo. Una ecografía transvaginal mostró la presencia de masas quísticas complejas de 5 centímetros de diámetro en ambos ovarios, multiloculadas, con un aspecto ultrasonográfico característico de endometriomas. Los marcado- res tumorales para neoplasia ovárica fueron negativos. Se programó una laparoscopia operatoria en la cual se determinó la imposibilidad técnica de realizar la anexo-histerectomía abdominal, por lo cual se descartó el tratamiento quirúrgico y se decidió iniciar tratamiento médico con 150 miligramos de acetato de medroxiprogesterona intramuscular. Un mes después, consultó refiriendo no haber tenido ninguna mejoría. Se propuso entonces el tratamiento con un endoceptivo de levonorgestrel (Mirena ${ }^{\circledR}$ ). La paciente aceptó esta posibilidad y ocho días después se aplicó el dispositivo de manera ambulatoria y sin dificultad. En las consultas de revisión al mes, tres y seis meses, la paciente refirió alivio total de sus síntomas y dijo sentirse satisfecha con su tratamiento.

\section{DISCUSIÓN}

El papel del endoceptivo de levonorgestrel en el tratamiento de la endometriosis parece ser promisorio. Se han publicado varias series de casos reportando mejoría significativa de los síntomas dolorosos de la endometriosis tras la aplicación de este implante intrauterino. Estos trabajos además, han evidenciado altos niveles de satisfacción por parte de las pacientes sometidas a este tratamiento. ${ }^{7-9}$

Uno de los reportes, demostró la disminución estadísticamente valorable del tamaño de nódulos endometriósicos presentes en el tabique rectovaginal de pacientes en quienes se hizo el diagnóstico y seguimiento por medio de ecografía transrectal. ${ }^{8}$ Un ensayo clínico aleatorizado reportó la disminución estadísticamente significativa de la dismenorrea y mayores niveles de satisfacción en el grupo de pacientes en quienes se aplicó un dispositivo liberador de levonorgestrel posterior a cirugía laparoscópica conservadora de endometriosis cuando se comparó con pacientes en quienes no se utilizó el endoceptivo después de la intervención quirúrgica. ${ }^{10}$

El seguimiento de una cohorte de pacientes con endometriosis y endoceptivo liberador de levonor- 
gestrel demostró una adhesión al tratamiento del $56 \%$ a los 3 años. ${ }^{11}$

Un ensayo clínico controlado y aleatorizado reportó una disminución estadísticamente significativa y comparable de los síntomas dolorosos de la enfermedad cuando uno de los grupos fue manejado con el endoceptivo de levonorgestrel y el otro con análogos de GnRH. ${ }^{12}$

Existen múltiples hipótesis de cómo el dispositivo intrauterino liberador de levonorgestrel puede afectar de manera benéfica el comportamiento de la endometriosis mejorando sus síntomas, entre ellos se mencionan: efecto directo del levonorgestrel sobre los receptores de estrógenos de los focos induciendo en ellos una reacción pseudodecidual; efecto directo sobre el endometrio induciendo atrofia con hipomenorrea o amenorrea como resultado del mismo; inhibición de mediadores inflamatorios en la pelvis y disminución del flujo sanguíneo pélvico con lo que mejorarían la hiperemia y la congestión del peritoneo generadas por la endometriosis. ${ }^{13}$

El reporte con los resultados satisfactorios sobre el uso del levonorgestrel por vía intrauterina en una paciente con pelvis congelada abre una nueva posibilidad de investigación y tratamiento para mujeres con contraindicación médica para la cirugía definitiva y que no tengan problemas para el uso de progestágenos; en mujeres que no estén seguras de querer practicarse un procedimiento quirúrgico agresivo o riesgoso o en aquellas pacientes en quienes, como en la nuestra, los riesgos quirúrgicos superen los posibles beneficios.

\section{CONCLUSIÓN}

El dispositivo intrauterino liberador de levonorgestrel se presenta como una opción de manejo médico a ser evaluada por medio de los diseños epidemiológicos adecuados en casos de endometriosis avanzada y sintomática, cuando una anexo-histerectomía por las condiciones de la paciente pueda resultar ser una opción riesgosa para su integridad.

\section{REFERENCIAS}

1. Winkel CA. Evaluation and management of women with endometriosis. Obstet Gynecol 2003;102:397-408.

2. Luciano DE, Luciano AA. Pain associated with endometriosis: therapeutic options. Women's Health 2006;2:617-26.

3. Hornstein MD, Surrey ES, Wesberg GW, Casino LA. Leuprolide acetate depot and hormonal add-back in endometriosis: a 12-month study. Lupron Add-Back Study Group. Obstet Gynecol 1998;91:16-24.

4. Vercellini P, Cortesi I, Crosignani PG. Progestins for symptomatic endometriosis: a critical analysis of the evidence. Fertil Steril 1997;68:393-491.

5. Namnoum AB, Hickman TN, Goodman SB, Gegkbach DL, Rock JA. Incidence of symptom recurrence after hysterectomy for endometriosis. Fertil Steril 1995;64:898-902.

6. Fedele L, Bianchi S, Raffaelli R, Portuese A, Dorta M. Treatment of adenomyosis-associated menorrhagia with levonorgestrel-releasing intrauterine device. Fertil Steril 1997;68:426-9.

7. Vercellini P, Aimi G, Panazza S, De Giorgi O, Pesole A, Crosignani PG. A levonorgestrel-releasing intrauterine system for the treatment of dysmenorrhoea associated with endometriosis: a pilot study. Fertil Steril 1999;72:505-8.

8. Fedele L, Bianchi S, Zanconato G, Portuese A, Rafaelli $\mathrm{R}$. Use of a levonorgestrel-releasing intrauterine device in the treatment of rectovaginal endometriosis. Fertil Steril 2001;75:485-8.

9. Lockhat FB, Emembolu JO, Konje JC. The evaluation of the effectiveness of an intrauterine administered progestogen (levonorgestrel) in the symptomatic treatment of endometriosis and in the staging of the disease. Hum Reprod 2004;19:179-84.

10. Vercellini P, Frontino G, De Giorgi O, Aimi G, Zaina B, Crosignani PG. Comparison of a levonorgestrel-releasing intrauterine device versus expectant management after conservative surgery for symptomatic endometriosis: a pilot study. Fertil Steril 2003;80:305-9.

11. Lockhat FB, Emembolu JO, Konje JC. The efficacy, side-effects and continuation rates in women with symptomatic endometriosis undergoing treatment with an intrauterine administered progestogen (levonorgestrel): a 3 year follow-up. Hum Reprod 2005:20:789-93. 
12. Petta CA, Ferriani RA, Abrao MS, Hassan D, Rosa E, Silva JC, et al. Randomized clinical trial of a levonorgestrel-releasing intrauterine system and a depot GnRH analogue for the treatment of chronic pelvic pain in women with endometriosis. Hum Reprod 2005;20:1993-8.
13. Lockhat FB, Emembolu JO, Konje JC. Serum and peritoneal fluid levels of levonorgestrel in women with endometriosis who were treated with an intrauterine contraceptive device containing levonorgestrel. Fertil Steril 2005;83:398-404.

Conflicto de intereses: En junio del 2006, el Doctor José Fernando De Los Ríos Posada, de manera independiente, presentó en el XXV Congreso Colombiano de Obstetricia y Ginecología la conferencia "Endocepción y Endometriosis" en un Simposio organizado por Laboratorios Schering, empresa productora y distribuidora de Mirena ${ }^{\circledR}$ en Colombia. A la fecha de publicación de este artículo, el Doctor De Los Ríos no tiene ningún vínculo laboral ni contractual con Laboratorios Schering. La elaboración y publicación de este trabajo han sido producto de su inquietud académica por el tema y no están motivadas por intereses económicos ni comerciales. 